\title{
STUDY OF ANXIETY, DEPRESSION AND PSYCHOSEXUAL DYSFUNCTION IN FUNCTIONAL GASTRODUODENAL DISORDERS
}

\author{
Tejaswini C. J1, Madhu Srinath², Shilpa Avarebeel ${ }^{3}$, Shivanand ${ }^{4}$
}

${ }_{1}^{1}$ Assistant Professor, Department of Medicine, JSS Academy of Research and Higher Education, Mysore, Karnataka, India.

${ }^{2}$ Associate Professor, Department of Community Medicine, JSS Academy of Research and Higher Education, Mysore, Karnataka, India.

${ }^{3}$ Senior Resident, Department of Medicine, JSS Academy of Research and Higher Education, Mysore, Karnataka, India.

${ }^{4}$ Assistant Professor, Department of Psychiatry, JSS Academy of Research and Higher Education, Mysore, Karnataka, India.

\section{ABSTRACT}

\section{BACKGROUND}

The association of anxiety, depression and psychosexual dysfunction in patients of functional gastroduodenal disorders has not been studied in detail among our population. There have always been mixed results with few studies favouring strong association with anxiety and few with depression and many more denying the presence of these psychiatric illnesses. There are also no data related to the psychosexual dysfunction in these patients.

The aim of this study was to estimate anxiety, depression and psychosexual issues in patients with functional gastroduodenal disorders and to determine the predominant gastroduodenal symptom in these patients.

\section{MATERIALS AND METHODS}

A total of 100 patients of either sex who had normal upper GI endoscopy and having symptoms suggestive of functional gastroduodenal disorders fulfilling Rome 4 criteria were evaluated for anxiety using HAM-A scale, depression using HAM-D scale and sexual disorders using ASEX scale and p values are calculated using chi-square test.

\section{RESULTS}

Post-prandial fullness, a symptom of functional gastroduodenal disorder was found in 55\% patients, of which 32 were women and 23 were men. Anxiety was seen in 71 people of whom $73.2 \%$ were women, depression was noted in 7 [15.9\%] men and 13 [23.2\%] women. Overall only $6 \%$ had no evidence of anxiety, depression or psychosexual issues. Psychosexual issues were seen in 3 [6.8\%] men and 16 [28.6\%] women.

\section{CONCLUSION}

It was observed that post-prandial fullness was the most predominant gastroduodenal complaint and anxiety was the most prevalent psychiatric issue. Depression was not statistically significant. Females than males had higher association of anxiety and sexual dysfunction with statistical significance.

\section{KEY WORDS}

Functional Dyspepsia, Post-prandial Fullness, Anxiety, Depression, Psychosexual Dysfunction.

HOW TO CITE THIS ARTICLE: Tejaswini CJ, Srinath M, Avarebeel S, et al. Study of anxiety, depression and psychosexual dysfunction in functional gastroduodenal disorders. J. Evolution Med. Dent. Sci. 2018;7(30):3385-3389, DOI: $10.14260 /$ jemds/2018/764

\section{BACKGROUND}

Clinicians encounter patients with symptoms suggestive of gastroduodenal disorder with no organic cause. These patients with functional gastroduodenal disorders do not benefit with conventional APD treatment. The association of anxiety, depression and psychosexual dysfunction with functional gastroduodenal disorders needs further clarity, as the results of several studies are conflicting. There are available reports which state that dyspepsia patients when investigated were found to be neurotic, anxious and depressed than non-dyspepsia controls. It is also suggested that depression, anxiety, phobia and somatisation are

'Financial or Other Competing Interest': None.

Submission 13-06-2017, Peer Review 08-07-2018,

Acceptance 13-07-2018, Published 23-07-2018.

Corresponding Author:

Dr. Tejaswini C. J,

No. 216, $3^{\text {rd }}$ Main, A1 Block,

Vijaynagar $3^{\text {rd }}$ Stage,

Mysore-570017,

Karnataka, India

E-mail: docteju27@gmail.com

DOI: $10.14260 /$ jemds $/ 2018 / 764$ strongly correlated with severity of dyspeptic symptoms. Nevertheless, there are many studies where there were no differences between patients with functional or organic dyspepsia in the prevalence or risk of mental distress. Furthermore, psychosexual issues which are a taboo to discuss in most communities are not studied in detail and there are no relevant studies to give us strong association.

\section{Aims and Objectives}

1. To estimate the proportion of patients with anxiety, depression and psychosexual dysfunction in patients with functional gastroduodenal disorders.

2. To compare the predominant gastroduodenal symptom/s in patients with anxiety, depression and psychosexual dysfunction.

\section{MATERIALS AND METHODS}

A cross-sectional study was conducted among hundred patients attending outpatient and inpatient Department of Medicine fulfilling the inclusion and exclusion criteria. Inclusion criteria was patients in the age group of 18 - 50 years, who had normal upper GI endoscopy and having symptoms suggestive of functional gastroduodenal disorders 
like post-prandial distress, epigastric pain, burning, early satiety, nausea, vomiting, belching and rumination fulfilling Rome 4 criteria, persisting for more than 3 months were included and evaluated.

Patients excluded were people who had an organic cause for their complaints, pregnant women, patients on chronic use of NSAIDS/ steroids and patients who had established chronic diseases like rheumatoid arthritis, connective tissue disease etc. and patients with DSM-IV AXIS psychiatric diagnosis.

Once the patient's gastroduodenal symptoms were recorded, they were subjected for psychiatric analysis through a questionnaire for anxiety using HAM-A scale, depression using HAM-D scale and sexual disorders using ASEX scale after obtaining a written consent from all patients.

The collected data was analysed using SPSS version 22.0. To compare the proportion of men and women with anxiety, depression and psychosexual dysfunction, Chi-square test was applied and the predominant symptom/s in these patients was analysed to see the prevalence and the proportion of anxiety, depression and psychosexual dysfunction in this set of patients was estimated.

\section{RESULTS}

Among 100 patients studied, there were 44 men and 56 women. The youngest person was 19 years of age and the oldest was 50 years of age and average being 38 years.

All the patients were married and were living with their spouses. When patients were analysed for their gastroduodenal complaints, it was found that post-prandial fullness was reported in 55 patients of whom 32 [57\%] were women and 23 [52\%] were men.

The second common symptom was epigastric burning reported in 31 patients followed by vomiting in 29 patients. There was a huge disparity in numbers when post-prandial fullness, belching and nausea were compared between men and women. It was observed that 32 women vs 23 men complained of post prandial fullness, 15 women vs 9 men complained of nausea and 13 women vs 7 men complained of belching. In our study early satiety was reported by 2 men and 5 women and rumination as a complaint was recorded in 5 patients, of which 3 were men and 2 were women. The results are presented in Figure 1.

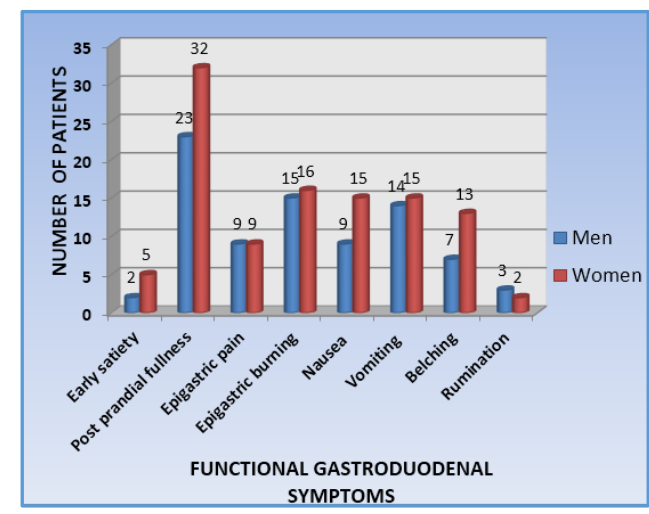

Figure 1. Proportions of patients with varied symptoms of Functional Gastroduodenal Disorders

After the initial assessment when the patients were subjected to psychiatric analysis with the HAM-A, HAM-D and ASEX score, we found the following results.
As depicted in Figure 2 HAM-A score revealed anxiety in 71 people, of which [30] 68.2\% were men and [41] $73.2 \%$ were women. HAM-D score recorded depression in 7 [15.9\%] men and 13 [23.2\%] women. Psychosexual issues were seen in 3 [6.8] men and 16 [28.6] women.

Our study showed anxiety in more number of patients than depression or psychosexual issues. These functional gastroduodenal patients who had association of psychiatric illness, the analysis of anxiety score and its relation to psychosexual dysfunction were tested and it is noteworthy to say that there was a significant positive correlation of 0.2 between the two.

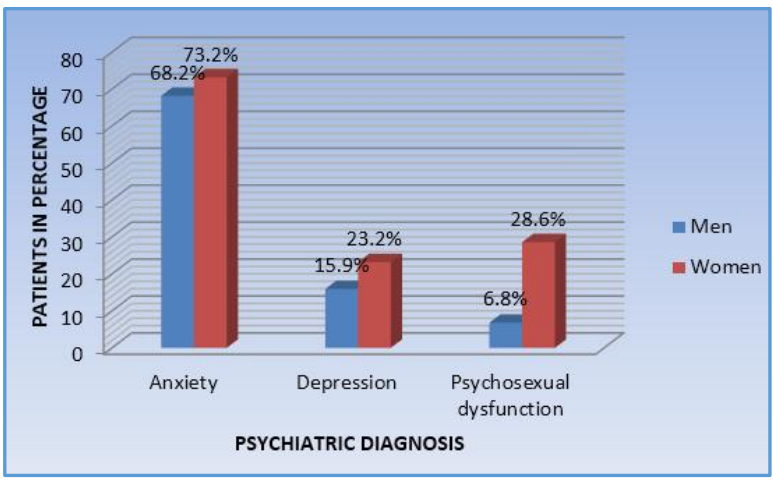

Figure 2. Comparison of proportion of patients with Anxiety, Depression and Psychosexual Dysfunction in Functional Gastroduodenal Disorder

\begin{tabular}{|c|c|c|c|}
\hline & Men & Women & P value \\
\hline Anxiety & $30[68.2]$ & $41[73.2]$ & 0.002 \\
\hline Depression & $7[15.9]$ & $13[23.2]$ & 0.512 \\
\hline Psychosexual Dysfunction & $3[6.8]$ & $16[28.6]$ & 0.008 \\
\hline $\begin{array}{c}\text { Table 1. Comparison of Anxiety, Depression and } \\
\text { Psychosexual Dysfunction in Functional Gastroduodenal } \\
\text { Disorder Patients }\end{array}$ \\
\hline
\end{tabular}

Table 1 shows that the prevalence of anxiety and sexual dysfunction was higher among women with functional gastroduodenal disorders when compared to men $[\mathrm{p}<$ 0.005].

Furthermore, individual symptom of functional gastroduodenal disorder was tested for their significant association with the psychiatric diagnosis. Majority of them had post-prandial fullness comprising of 23 men and 32 women.

For further clarity, patients with post-prandial fullness [the predominant complaint] were analysed for their interrelation with anxiety, depression and psychosexual dysfunction.

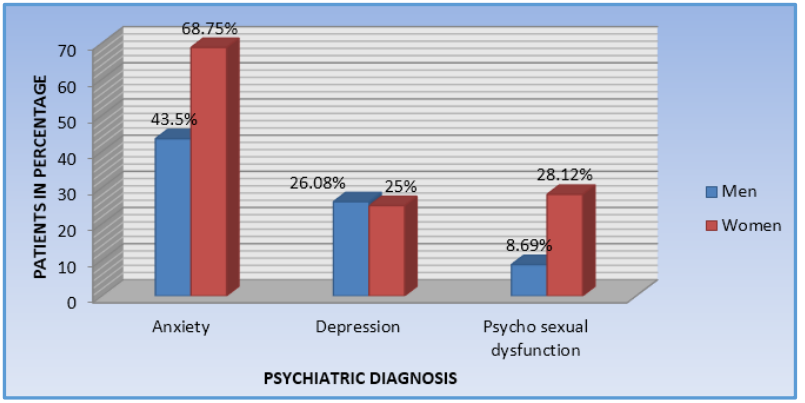

Figure 3. Comparison of Anxiety, Depression and Psychosexual Dysfunction in patients of Post-Prandial Fullness Syndrome 


\begin{tabular}{|c|c|c|c|}
\hline & Men & Women & p value \\
\hline Anxiety & $10[43.5 \%]$ & $22[68.75 \%]$ & 0.002 \\
\hline Depression & $6[26.08 \%]$ & $8[25 \%]$ & 0.221 \\
\hline $\begin{array}{c}\text { Psychosexual } \\
\text { dysfunction }\end{array}$ & $2[8.69 \%]]$ & $9[28.12 \%]]$ & 0.008 \\
\hline
\end{tabular}

Table 2. Comparison of Anxiety, Depression and Psychosexual Dysfunction in patients of Post-prandial Fullness

As per Figure 3, among the 23 men with post-prandial fullness there were 10 men who had anxiety and among 32 females there were 22 women with anxiety. 6 men and 8 women of post-prandial fullness syndrome had depression. And 2 men and 9 women with post-prandial fullness also had psychosexual dysfunction. Table 2 shows p-value being significant with anxiety and psychosexual dysfunction, but not with depression compared between the two sexes.

\section{DISCUSSION}

Functional gastrointestinal disorders are group of disorders, in which patients have chronic symptoms that can be ascribed to the gastroduodenal region. A classification of the functional gastroduodenal disorders is proposed by Rome to simplify the diagnosis. Correspondingly, four categories of functional gastroduodenal disorders are recognised. The first category, functional dyspepsia sorts patients with symptoms such as epigastric pain or burning, post-prandial fullness or early satiation. Recently, there are evidences and ongoing clinical experience that has unravelled a subgroup classification for post-prandial distress syndrome (early satiation or post-prandial fullness) and epigastric pain syndrome (pain or burning in the epigastrium). The second category in the Rome classification are the belching disorders, which comprises aerophagia (troublesome repetitive belching with observed excessive air swallowing) and unspecified belching (no evidence of excessive air swallowing). The third category, nausea and vomiting disorders comprises chronic idiopathic nausea (frequent bothersome nausea without vomiting), functional vomiting (recurrent vomiting in the absence of self-induced vomiting or underlying eating disorders, metabolic disorders, drug intake or psychiatric or central nervous system disorders) and cyclic vomiting syndrome (stereotypical episodes of vomiting with vomiting-free intervals). The rumination syndrome is a fourth category of functional gastroduodenal disorder characterised by effortless regurgitation of recently ingested food into the mouth followed by re-chewing and reswallowing or expulsion. Multiple studies have shown an association between anxiety and depression with functional gastrointestinal symptoms, but none have established the direction of association or causality.

Psychosocial factors have well-known association in pathogenesis of functional gastroduodenal disorders. It is still speculated whether these play a pivotal role in the pathophysiology of the symptom complex. It is also hypothesised that they may contribute to a common susceptibility for functional and psychological disorders. ${ }^{1}$ In patients with functional gastroduodenal disorders, it is found that functional dyspepsia is the most common diagnosis. When a psychiatric diagnosis is speculated in these patients, a large scale epidemiologic study showcased that anxiety is more common in patients with the diagnosis of FD; however, the same group did not have a higher depression score. ${ }^{2}$ Another recent large-scale cohort study of 1175 patients showed that among people who were free of FD at baseline were followed up for a period of 12 years and later found that higher levels of anxiety but not depression at baseline was a significant independent predictor of developing new-onset functional dyspepsia. ${ }^{3}$ The same set of patients when further probed it was found that in patients of functional dyspepsia the complaint of post-prandial fullness was the common grievance, but these findings have not been consistent in other studies which makes it further difficult to conclude. Not merely stating the diagnosis of functional dyspepsia, a recent study examined the correlation between subtypes of FD with psychiatric abnormalities and personality traits. Postprandial distress syndrome was independently associated with somatisation (correlation coefficient: 0.28), depression (correlation coefficient: 0.27) and phobia (correlation coefficient: 0.24), while epigastric pain syndrome was not significantly correlated with any psychiatric abnormality. ${ }^{4}$ It is still unclear why meal-related symptoms (post-prandial distress syndrome) are associated with anxiety and not with epigastric pain or burning (epigastric pain syndrome) and needs further investigation. This observation certainly suggests that symptoms are unlikely to be driving the development of anxiety in functional dyspepsia. It is because it then would be expected that pain rather than discomfort would induce more anxiety. Somatisation has also been linked to the prevalence and severity of FD symptoms in other studies as well.5,6 The underlying mechanisms of why there are meal-related symptoms may include the pathophysiology of fundic disaccommodation and visceral hypersensitivity, but whether these abnormalities are centrally mediated is uncertain. In a study of 201 tertiary care patients with functional dyspepsia, the symptom severity was determined largely by somatisation, 2,6 which may in turn be genetically driven. The search for common pathways that induce anxiety and dyspepsia now needs greater attention. ${ }^{7}$ The other study which supported the above findings was done by David J et al, who described that anxiety had a positive correlation with functional dyspepsia which is in consensus with our findings. His views were similar to the Kalixanda study which found anxiety, but not depression to be associated with dyspepsia. ${ }^{8}$ Nevertheless, findings are not universally accepted by all authors. Holtman et al describes patients with functional dyspepsia have some genotypic similarity with patients with depression and found depression in many patients with functional gastrointestinal disorders. It has been shown that homozygous G-protein beta 3 C825T polymorphism is associated with functional dyspepsia and is linked to depression which favours a community-based study, which states a strong association between major depressive episode and dyspepsia, even higher than that for generalised anxiety. ${ }^{9}$ However, our study results were not supportive with this information, as the diagnosis of depression according to the questionnaire was not statistically significant in our population with functional gastroduodenal disorders.

The other symptoms of functional gastroduodenal disorder were not very common among our population. It is very uncommon to have association of chronic nausea without vomiting. But when patients have vomiting in the absence of nausea, it becomes more ominous sign of raised 
intracranial pressure and might prompt a suspicion of an organic central nervous system disease. Nausea may be mealrelated or unrelated, suggesting potential pathogenic heterogeneity. In earlier studies done elsewhere when patients of chronic nausea and vomiting were analysed, the severity indices of the symptoms were similar between groups tested for nausea, retching, vomiting and also there were no significant differences among the subjects in health care utilisation, quality of life indices, depression or trait anxiety scores. However, comparing the psychiatric diagnosis, anxiety scores were slightly higher among patients with delayed gastric emptying. Progressive analysis of the other symptoms of functional gastroduodenal disorder, it is noted that belching does not always arise from swallowing air. Availability of sophisticated methods like high-resolution manometry and impedance monitoring system enables the objective differentiation of supragastric belching from gastric belching and this has helped to draw an inference that supragastric belching is more often a habituation of laryngeal air release than the patient actual swallowing air. Our study did not go into these differentiating details, as the number of people with this significant complaint was negligible. But the other finding which was striking among our patients was that nausea, belching and vomiting was seen more in females, but even then, it failed to establish a statistical significance. Rumination is characterised by the voluntary or involuntary regurgitation and re-chewing of partially digested food, that is either re-swallowed or expelled. This regurgitation appears effortless, may be preceded by a belching sensation and typically does not involve retching or nausea. In rumination, the regurgitant does not taste sour or bitter. While the pathophysiology of rumination remains unclear, a proposed mechanism is that in patients with the above complaint whenever there is gastric distention with food it is followed by abdominal compression and relaxation of the lower oesophageal sphincter. These actions allow stomach contents to be regurgitated and re-chewed and then swallowed or expelled. In our study rumination was not a common symptom and it was expressed in few females, but it could not help in forming a statistically significant symptom. Few studies noted that rumination was found to be associated not only with depressive symptoms, but also with both types of anxiety. Finally, the type of rumination did not significantly predict depressive symptoms, somatic anxiety or trait anxiety.

The uncertainty surrounding the pathology and treatment aspects push many patients with dyspepsia to try out alternative therapies, which are totally of unproven value. ${ }^{10}$ Non-pharmacological treatments have also been tested, but only in very limited numbers. It was noted that hypnotherapy was superior to standard care in a recent single-centre study, but unfortunately it is not widely available. ${ }^{11}$ Adding to the confusion, a systematic review concluded that psychological therapy in functional dyspepsia is not of established value, because of limited data.12 Psychological treatment also represents a labour-intensive and costly approach. Even after a number of studies done to establish the physiology and associations, the lack of effective management in functional dyspepsia is expected to promote repeated medical consultation and its associated costs and also the substantial amounts of time lost from work.13,14,15 There are not many studies discussing the association of psychosexual issues in functional gastroduodenal disorders. A few studies which have noted the prevalence of self-reported sexual dysfunction in patients with functional GI disorders has recorded $43.3 \%$ and this did not differ by gender, age stratification or disease subtype like Irritable Bowel Syndrome (IBS), Non-Ulcer Dyspepsia (NUD) or functional dyspepsia or patients having both. In the comparison which is done so far in patients of irritable bowel syndrome, subjects who seek medical aid for sexual dysfunction and in those who do not the reported sexual dysfunction prevalence was significantly lower (16.1 and $24.4 \%$ respectively, $\mathrm{p}<0.005$ ). Specific analysis of the symptomatology reveals decreased sexual drive was the symptom most commonly reported by both male $(36.2 \%)$ and female $(28.4 \%)$ patients. Dyspareunia was reported by $16.4 \%$ of females and $4 \%$ of males with IBS, but was rarely observed in patients with functional dyspepsia. Report of sexual dysfunction was positively associated with perceived GI symptom severity, but not with psychological symptom severity. ${ }^{16}$ Our analysis show that not many men had statistically significant psychosexual issues associated with their gastroduodenal symptoms, but females who on the other hand had positive psychosexual issues in $28.6 \%$ which also had an impressive correlation with anxiety accounting to 0.27 . There have been no prior studies or reports, which gave any insight to this finding and require further evaluation in a larger population to support our results.

\section{CONCLUSION}

Functional gastroduodenal disorders have a strong association with psychiatric illness. Post-prandial fullness was the most common symptom in patients with functional gastroduodenal disorder and females outnumbered men with this as a presenting complaint. Psychiatric analysis of this cohort of patient's revealed anxiety was the most prevalent issue and depression was not statistically significant in our patients. Anxiety and psychosexual issues together had a positive correlation and it was also noted that psychosexual issues and anxiety were statistically higher in females when compared to males with functional gastroduodenal disorder.

\section{REFERENCES}

[1] Tack J, Talley NJ, Camilleri M, et al. Functional gastroduodenal disorders. Gastroenterology 2006;130(5):1466-79.

[2] Aro P, Talley NJ, Ronkainen J, et al. Anxiety is associated with uninvestigated and functional dyspepsia (Rome III criteria) in a Swedish populationbased study. Gastroenterology 2009;137(1):94-100.

[3] Bensoussan A, Talley NJ, Hing M, et al. Treatment of irritable bowel syndrome with Chinese herbal medicine: a randomized controlled trial. Jama 1998;280(18):1585-9.

[4] Tack J, Talley NJ, Camilleri M, et al. Functional gastroduodenal disorders. Gastroenterology 2006;130(5):1466-79.

[5] Hsu YC, Liou JM, Liao SC, et al. Psychopathology and personality trait in subgroups of functional dyspepsia based on Rome III criteria. The American Journal of Gastroenterology 2009;104(10):2534-42. 
[6] Castillo EJ, Camilleri M, Locke GR, et al. A communitybased, controlled study of the epidemiology and pathophysiology of dyspepsia. Clinical Gastroenterology and Hepatology 2004;2(11):985-96.

[7] Van Oudenhove L, Vandenberghe J, Geeraerts B, et al. Determinants of symptoms in functional dyspepsia: gastric sensorimotor function, psychosocial factors or somatisation? Gut 2008;57(12):1666-73.

[8] Camilleri CE, Carlson PJ, Camilleri M, et al. A study of candidate genotypes associated with dyspepsia in a US community. The American Journal of Gastroenterology 2006;101(3):581-92.

[9] Ronkainen J, Aro P, Storskrubb T, et al. Gastro-oesophageal reflux symptoms and health-related quality of life in the adult general population-the Kalixanda study. Alimentary Pharmacology \& Therapeutics 2006;23(12):1725-33.

[10] Holtzman CW, Trotman HD, Goulding SM, et al. Stress and neurodevelopmental processes in the emergence of psychosis. Neuroscience 2013;249:172-91.
[11] Calvert EL, Houghton LA, Cooper P, et al. Long-term improvement in functional dyspepsia using hypnotherapy. Gastroenterology 2002;123(6):177885.

[12] Soo S, Moayyedi P, Deeks J, et al. Psychological interventions for non-ulcer dyspepsia. Cochrane Database Syst Rev 2005;(2):CD002301.

[13] Locke GR 3rd. The epidemiology of functional gastrointestinal disorders in North America. Gastroenterology Clinics of North America 1996;25(1):1-19.

[14] Sandler RS, Everhart JE, Donowitz M, et al. The burden of selected digestive diseases in the United States. Gastroenterology 2002;122(5):1500-11.

[15] Talley NJ, Silverstein MD, Agreus L, et al. AGA technical review: evaluation of dyspepsia. American Gastroenterological Association 1998;114(3):582-95.

[16] Fass R, Fullerton S, Naliboff B, et al. Sexual dysfunction in patients with irritable bowel syndrome and nonulcer dyspepsia. Digestion 1998;59(1):79-85. 